\title{
COMPATIBLE TIGHT RIESZ ORDERS ON ORDERED PERMUTATION GROUPS
}

\author{
G. DAVIS and E. LOCI*
}

(Received 27 September 1974; revised 17 February 1975)

\begin{abstract}
The pointwise order makes the group $A(\Omega)$ of order-preserving permutations of a totallyordered set $\Omega$ a lattice-ordered group. We give some criteria for determining the compatible tight Riesz orders on $A(\Omega)$ in the case of $\Omega$ being a totally-ordered field, and then obtain various adjunctions... one between tight Riesz orders on $A(\Omega)$ and certain ideals of the fixed point lattice $\Phi(\Omega)$, and a second between maximal tangents and certain filters of $\Phi(\Omega)$. We also establish a correspondence between tight Riesz orders and first-order properties. Finally, we make use of our results to say what we can in the case of the automorphisms of the real field, and to pose several open problems.
\end{abstract}

\section{Introduction}

We start from a theorem due essentially to Wirth (1973), which states that a compatible tight Riesz order on a lattice-ordered group $(G, \leqslant)$ is determined by a subset $T$ of the positive set $G^{+}=\{x \in G: 1 \leqslant x\}$ of $G$ satisfying the following conditions:

(1) $T$ is a proper dual ideal of $G^{+}$

(2) $T$ is normal in $G$

(3) $T=T \cdot T$

(4) $1 \leqslant x^{n} \leqslant y$ for all positive integers $n$, for all $y \in T$, implies $x=1$. Condition (4) can be replaced by the equivalent condition

(4') $\wedge_{x \in T} x=1$.

Any compatible tight Riesz order $\leqq$ on $G$ has a strict positive set $T=\{x \in G: 1<x\}$ satisfying (1)-(4), and conversely any such set $T$ is the strict positive set for a compatible tight Riesz order on $G$. We sometimes say, by an abuse of language, that such a set $T$ is a compatible tight Riesz order on $G$.

The group $A(\Omega)$ of order-preserving permutations of a totally-ordered set $\Omega$ is a lattice-ordered group when ordered by $\pi \geqslant 1$ if $\pi(x) \geqq x$ in $\Omega$ for all $x \in \Omega$.

* Formerly, E. BOLZ. 
Our main problem is to determine the compatible tight Riesz orders on $A(\Omega)$. Throughout this paper we will assume that $\Omega$ is a totally-ordered field. The reasons for this are roughly as follows: the condition (3) for the strict positive set for a compatible tight Riesz order is generally the most difficult to check but follows easily if the group is divisible, and we know $A(\Omega)$ is divisible when $\Omega$ is a totally-ordered field. Furthermore most of our results are vaccuous if the set $T_{0}=\{\pi \in A(\Omega): \pi(x)>x$ for all $x \in \Omega\}$ is empty, and if $\Omega$ is a totally-ordered field then $x \rightarrow x+1$ belongs to $T_{0}$.

We are grateful to Colin Fox and Arthur Jones for their assistance, and especially grateful to the referee for his helpful suggestions, and for providing an alternative proof of Proposition 14.

\section{Maximal tangents}

For $\pi \geqslant 1$ in $A(\Omega)$ we define $\operatorname{Supp}(\pi)=\{x \in \Omega: \pi(x)>x\}$. This is the complement of the set $\operatorname{Fix}(\pi)=\{x \in \Omega: \pi(x)=x\}$ of fixed points of $\pi$. The collection $\{\operatorname{Supp}(\pi): \pi \geqslant 1\}$, ordered by inclusion, is a lattice as the formulae

$$
\begin{aligned}
& \operatorname{Supp}\left(\pi_{1}\right) \cap \operatorname{Supp}\left(\pi_{2}\right)=\operatorname{Supp}\left(\pi_{1} \wedge \pi_{2}\right) \\
& \operatorname{Supp}\left(\pi_{1}\right) \cup \operatorname{Supp}\left(\pi_{2}\right)=\operatorname{Supp}\left(\pi_{1} \vee \pi_{2}\right)
\end{aligned}
$$

for $\pi_{1}, \pi_{2} \geqslant 1$, show.

Lemma 1. If $\pi \geqslant 1$ in $A(\Omega)$ then there exist $\pi_{i}, \pi_{2} \in A(\Omega)$ such that $\pi=\pi_{1} \cdot \pi_{2}, \pi_{i} \geqslant 1$ and $\operatorname{Supp}\left(\pi_{i}\right)=\operatorname{Supp}(\pi)(i=1,2)$.

This follows since $A(\Omega)$ is divisible.

Proposition 2. For any totally-ordered field $\Omega$ the lattice-ordered group $A(\Omega)$ admits at least four distinct compatible tight Riesz orders. Specifically,

$$
\begin{aligned}
& T_{0}=\{\pi \in A(\Omega): \pi(x)>x \text { for all } x \in \Omega\} \\
& T_{\alpha}=\left\{\pi \in A^{+}(\Omega): \text { for some } y \in \Omega, \pi(x)>x \text { for all } x \geqq y\right\} \\
& T_{\beta}=\left\{\pi \in A^{+}(\Omega): \text { for some } y \in \Omega, \pi(x)>x \text { for all } x \leqq y\right\} \\
& T_{\alpha \beta}=T_{\alpha} \cap T_{\beta}
\end{aligned}
$$

are strict positive sets for distinct compatible tight Riesz orders on $A(\Omega)$.

Proof $T_{0}$. This set is clearly a proper dual ideal of $A^{+}(\Omega)$ that is normal in $A(\Omega)$. The inclusion $T_{0} \cdot T_{0} \subseteq T_{0}$ is obvious and the reverse inclusion follows from Lemma 1 . Since $A(\Omega)$ is divisible condition (4) is equivalent to $\pi_{0}=1$ for any $\pi_{0}$ satisfying $1 \leqslant \pi_{0} \leqslant \pi$ for all $\pi \in T_{0}$. If $1 \leqslant \pi_{0} \leqslant \pi$ for all $\pi \in T_{0}$, but $\pi_{0} \neq 1$ then $\pi_{0}(x)>x$ for some $x \in \Omega$. We define $\pi_{1} \in A(\Omega)$ by $\pi_{1}(z)=$ $z+\frac{1}{2}\left(\pi_{0}(x)-x\right)$ and then $\pi_{1} \in T_{0}$ but $\pi_{1}(x)=\frac{1}{2}\left(\pi_{0}(x)+x\right)<\pi_{0}(x)$, which contradicts $\pi_{0} \leqslant \pi_{1}$. Therefore $\pi_{0}=1$. 
The remaining sets $T_{\alpha}, T_{\beta}, T_{\alpha \beta}$ are easily seen to satisfy (1)-(3), and being larger than $T_{0}$ they satisfy (4).

A subset $P$ of $A(\Omega)$ is a prime subgroup of $A(\Omega)$ if $P$ is a convex sublattice subgroup of $A(\Omega)$ for which $\pi_{1} \wedge \pi_{2}=1$ implies that either $\pi_{1} \in P$ or $\pi_{2} \in P$.

For a compatible tight Riesz order $T$ on $A(\Omega)$, Reilly (1973), (to appear) shows that if $P$ is a prime subgroup of $A(\Omega)$ meeting $T$ then $P$ is convex for the order $\leqq$ defined by $T$, and the set $R(P)$ of right cosets of $P$ is totally-ordered for the canonical order induced from $\leqq$ on $A(\Omega)$, and if $P$ is maximal with respect to being a convex sublattice subgroup not meeting $T$ then $R(P)$ is totally-ordered and dense for the canonical order induced from $\leqq$ on $A(\Omega)$. The convex sublattice subgroups of $A(\Omega)$ that are maximal with respect to not meeting $T$ are prime subgroups and they have been called the maximal tangents of $T$ by Miller (1973).

A standard Zorn's lemma argument shows that any convex sublattice subgroup not meeting a compatible tight Riesz order $T$ is contained in a maximal tangent of $T$.

We define the fixed-point lattice, $\Phi(\Omega)$, of $\Omega$ to be the set $\{$ Fix $(\pi): \pi \geqslant 1$ \} ordered by inclusion. We see that since $\Omega$ is a totally-ordered field the fixed-point lattice of $\Omega$ contains $\Omega$ : for every $x \in \Omega$ there is a (piecewise linear) $\pi \in A(\Omega)$ with $\pi \geqslant 1$ and $\operatorname{Fix}(\pi)=\{x\}$.

For $x \in \Omega$ the stabilizer subgroup of $x$ is $P_{x}=\{\pi \in A(\Omega): \pi(x)=x\}$. We can also define stabilizer subgroups of points $\xi$ of the Dedekind completion $\bar{\Omega}$ of $\Omega$ : firstly every $\pi \in A(\Omega)$ can be extended to a $\bar{\pi} \in A(\bar{\Omega})$ by $\bar{\pi}(\xi)=$ $\sup \{\pi(x): x \in \Omega, x \leqq \xi\}$, and then we define $P_{\xi}=\{\pi \in A(\Omega): \bar{\pi}(\xi)=\xi\}$. McClearly (1969) has shown that the $P_{\xi}, \xi \in \bar{\Omega}$, are precisely the join-closed prime subgroups of $A(\Omega)$. We note that for $x \in \Omega, P_{x}$ is a prime subgroup not meeting $T_{0}$. However for $\xi \in \bar{\Omega} \mid \Omega$ we can have $P_{\xi} \cap T_{0} \neq \square$. This happens, for instance, when $\Omega$ is the field of rational numbers (i.e. the prime subfield of any totally-ordered field) since we can take a strictly increasing sequence $\left\{x_{n}: n \in N\right\}$ and a strictly decreasing sequence $\left\{y_{n}: n \in N\right\}$, both in $\Omega$, with $\sup \left\{x_{n}: n \in\right.$ $N\}=\sqrt{2}=\inf \left\{y_{n}: n \in N\right\}$ and then find a piecewise linear $\pi \in A(\Omega)$ whose graph contains the points $\left\{\left(x_{n}, x_{n+1}\right): n \in N\right\}$ and the points $\left\{\left(y_{n+1}, y_{n}\right): n \in N\right\}$ in $\Omega \times \Omega$. We then have $\pi(x)>x$ for all $x \in \Omega$ but $\bar{\pi}(\sqrt{2})=\sqrt{2}$.

\section{Proposition 3. For every $x \in \Omega, P_{x}$ is a maximal tangent of $T_{0}$.}

Proof. If $P_{x}$ is not a maximal tangent of $T_{0}$ then $P_{x}$ is properly contained in a maximal tangent $M$ of $T_{0}$. That is, $\pi_{0} \in M \backslash P_{x}$ for some $\pi_{0} \geqslant 1$. Let $\pi$ be an element of $P_{x}$ with Fix $(\pi)=\{x\}$. Then Fix $\left(\pi_{0} \vee \pi\right)=\operatorname{Fix}\left(\pi_{0}\right) \cap \operatorname{Fix}(\pi)=\square$ so $\pi_{0} \vee \pi \in T_{0} \cap M$ and this contradicts $T_{0} \cap M=\square$.

We now consider basic adjunctions that relate tight Riesz orders $T$ on 
$A(\Omega)$ to certain ideals $I$ of the fixed-point lattice $\Phi(\Omega)$, and maximal tangents of $T$ to filters of $\Phi(\Omega)$ maximal with respect to not meeting $I$.

We say that a subset $T$ of $A(\Omega)$ satisfying (1)-(3) of the introduction is a tight Riesz order on $A(\Omega)$ (as distinct from a compatible tight Riesz order on $A(\Omega)$ ) and that a lattice ideal $I$ of $\Phi(\Omega)$ is an $A(\Omega)$-invariant ideal if $I \neq \Phi(\Omega)$ and $\pi(\xi) \in I$ for all $\xi \in I$ and all $\pi \in A(\Omega)$. We use the term adjunction (c.f. Maclane [3]) instead of the equivalent "dual Galois Correspondence" i.e. an adjunction $f \mid-g$ between partially ordered sets $P$ and $Q$ is a pair of order preserving mappings $f: P \rightarrow Q, g: Q \rightarrow P$ such that $x \leqslant g(y)$ in $P$ iff $f(x) \leqslant y$ in $Q$ and then $x \in P$ is an algebra if and only if $g \circ f(x)=x$.

THEOREM 4. There is an adjunction $f \mid-g$ from the set of tight Riesz orders on $A(\Omega)$ ordered by inclusion, to the set of $A(\Omega)$-invariant ideals of $\Phi(\Omega)$, ordered by inclusion, such that the algebras for this adjunction are just the tight Riesz orders $T$ for which $\pi_{0} \in T$ implies $\left\{\pi \in A^{+}(\Omega): \operatorname{Supp}(\pi)=\operatorname{Supp}\left(\pi_{0}\right)\right\} \subseteq T$.

Proof. Let $T$ be a tight Riesz order on $A(\Omega)$. The subset $I=$ $\{$ Fix $(\pi): \pi \in T\}$ of $\Phi(\Omega)$ is closed under the formation of finite joins since $\pi_{1}$, $\pi_{2} \in T$ implies Fix $\left(\pi_{1}\right) \cup \operatorname{Fix}\left(\pi_{2}\right)=\operatorname{Fix}\left(\pi_{1} \wedge \pi_{2}\right) \in I$. Further, if Fix $\left(\pi_{0}\right) \in I$ and $\pi \in A(\Omega)$ then $\pi\left(\operatorname{Fix}\left(\pi_{0}\right)\right)=\operatorname{Fix}\left(\pi \pi_{0} \pi^{-1}\right) \in I$ since $T$ is normal in $A(\Omega)$. The ideal generated by $I$, viz. $\left\{\operatorname{Fix}(\pi)\right.$ : Fix $(\pi) \subseteq \operatorname{Fix}\left(\pi^{\prime}\right)$ for some $\left.\pi^{\prime} \in T\right\}$ is then an $A(\Omega)$-invariant ideal of $\Phi(\Omega)$ which we denote by $f(T)$. The mapping $T \rightarrow f(T)$ is clearly order-preserving. Conversely, if $I$ is an $A(\Omega)$-invariant ideal of $\Phi(\Omega)$ we define $g(I)=\left\{\pi \in A^{+}(\Omega)\right.$ : Fix $\left.(\pi) \in I\right\}$. Then, as above, $g(I)$ is a proper subset of $A^{+}(\Omega)$ closed under the formation of finite meets and is normal in $A(\Omega)$. Further, if $\pi_{0} \in g(I)$ and $\pi \geqslant \pi_{0}$ then Fix $(\pi) \subseteq$ Fix $\left(\pi_{0}\right) \in I$ so $\pi \in g(I)$. That is, $g(I)$ is a tight Riesz order on $A(\Omega)$. It is then straightforward to verify that for a tight Riesz order $T$ and an $A(\Omega)$-invariant ideal $I, f(T) \subseteq I$ if and only if $T \subseteq g(I)$, so that $f$ is a left-adjoint for $g$. The algebras for this adjunction are just the tight Riesz orders $T$ satisfying $T=g(f(t))$, and since Fix $(\pi)=\Omega \mid \operatorname{Supp}(\pi)$ for $\pi \geqslant 1$, these are just the tight Riesz orders $T$ such that $\pi_{0} \in T$ implies $\left\{\pi \in A^{+}(\Omega): \operatorname{Supp}(\pi)=\operatorname{Supp}\left(\pi_{0}\right)\right\} \subseteq T$.

We say that a tight Riesz order $T$ on $A(\Omega)$ is algebraic if $T$ is an algebra for the adjunction of Theorem 4.

COROLlaRy 5. Every algebraic tight Riesz order on $A(\Omega)$ is a compatible tight Riesz order.

Proof. The algebraic tight Riesz orders on $A(\Omega)$ are in one-one orderpreserving correspondence with the $A(\Omega)$-invariant ideals of $\Phi(\Omega)$. The smallest such ideal is $\{\square\}$ and the corresponding tight Riesz order is $T_{0}$ which is compatible, so all other algebraic tight Riesz orders are compatible. 
COROLLARY 6. Every maximal tight Riesz order on $A(\Omega)$ is algebraic and therefore a compatible tight Riesz order.

THEOREM 7. If $T$ is an algebraic tight Riesz order on $A(\Omega)$ there is an adjunction $f^{T} \mid-g^{T}$ from the set of convex sublattice subgroups of $A(\Omega)$ not meeting $T$, ordered by inclusion, to the set of filters of $\Phi(\Omega)$ not meeting $f(T)$, ordered by inclusion, such that the algebras for this adjunction are just the convex sublattice subgroups $G$ of $A(\Omega)$ not meeting $T$ for which $\pi_{0} \in G \cap$ $A^{+}(\Omega)$ implies $\left\{\pi \in A^{+}(\Omega)\right.$ : $\left.\operatorname{Supp}(\pi)=\operatorname{Supp}\left(\pi_{0}\right)\right\} \subseteq G$.

Proof. Let $T$ be an algebraic tight Riesz order and $G$ a convex sublattice subgroup of $A(\Omega)$ not meeting $T$. The subset $F=\left\{\operatorname{Fix}(\pi): \pi \in G \cap A^{+}(\Omega)\right\}$ is then closed under the formation of finite meets. If Fix $(\pi) \in F \cap f(T)$ where $\pi \in G$ then $\operatorname{Fix}(\pi) \subseteq \operatorname{Fix}\left(\pi^{\prime}\right)$ for some $\pi^{\prime} \in T$, so that $\operatorname{Fix}(\pi)=$ Fix $(\pi) \cap \operatorname{Fix}\left(\pi^{\prime}\right)=\operatorname{Fix}\left(\pi \vee \pi^{\prime}\right)$ which gives $\pi \in T \cap G$ since $T$ is algebraic and $\pi \vee \pi^{\prime} \in T$, a contradiction. Thus the filter $f^{T}(G)$ of $\Phi(\Omega)$ generated by $F$ does not meet $f(T)$. Conversely if $F$ is a filter of $\Phi(\Omega)$ not meeting $f(T)$ then $H=\left\{\pi \in A^{+}(\Omega)\right.$ : Fix $\left.(\pi) \in F\right\}$ is a convex sublattice of $A^{+}(\Omega)$ not meeting $T$. Further, $H$ is a subsemigroup of $A^{+}(\Omega)$ since Fix $\left(\pi_{1} \cdot \pi_{2}\right) \supseteq \operatorname{Fix}\left(\pi_{1}\right) \cap$ Fix $\left(\pi_{2}\right)$ for all $\pi_{1}, \pi_{2} \in A^{+}(\Omega)$. The subgroup $g^{T}(F)$ of $A(\Omega)$ generated by $H$ is therefore a convex sublattice subgroup of $A(\Omega)$ not meeting $T$. For convex sublattice subgroups $H_{1}, H_{2}$ of $A(\Omega)$ we have $H_{1} \subseteq H_{2}$ if and only if $H_{1} \cap A^{+}(\Omega) \subseteq H_{2} \cap A^{+}(\Omega)$. This allows us to see, as in Theorem 4, that $f^{T}(G) \subseteq F$ if and only if $G \subseteq g^{T}(F)$ when $G$ is a convex sublattice subgroup of $A(\Omega)$ not meeting $T$ and $F$ is a filter of $\Phi(\Omega)$ not meeting $f(T)$. The statement about the algebras for this adjunction follows readily.

$\dot{W}$ e say that a tight Riesz order $T$ is prime if $\pi_{1}, \pi_{2} \in A^{+}(\Omega)$ and $\pi_{1} \vee \pi_{2} \in T$ implies that either $\pi_{1} \in T$ or $\pi_{2} \in T$. If $T$ is an algebraic tight Riesz order then $T$ is prime if and only if $f(T)$ is a prime ideal of the distributive lattice $\Phi(\Omega)$.

PROPOSITION 8. If $T$ is a maximal, and hence algebraic, tight Riesz order on $A(\Omega)$ then $T$ has a unique maximal tangent $M$ exactly when $T$ is prime. In this case $M$ is normal in $A(\Omega)$ so that $A(\Omega) / M$ is a totally-ordered group.

Proof. If $T$ is prime then $f(T)$ is a prime $A(\Omega)$-invariant ideal of $\Phi(\Omega)$ so that $\Phi(\Omega) \backslash f(T)$ is the unique filter maximal with respect to not meeting $f(T)$. Further $\Phi(\Omega) \backslash f(T)$, as the complement of $f(T)$, is $A(\Omega)$-invariant, so the maximal tangent $M$ corresponding to $\Phi(\Omega) \backslash f(T)$ is the unique maximal tangent of $T$ and $M$ is normal in $A(\Omega)$. Conversely, if $T$ is not prime then $f(T)$ is not prime in $\Phi(\Omega)$ so there are at least two filters maximal with respect to not meeting $f(T)$, and thus $T$ has at least two maximal tangents. 


\section{Compactifications}

We furnish $\Omega$ with the order topology which has the sets

$$
\begin{aligned}
(-\infty, a) & =\{x \in \Omega: x<a\} \\
(a, b) & =\{x \in \Omega: a<x<b\} \\
(a, \infty) & =\{x \in \Omega: a<x\}
\end{aligned}
$$

as a join-base for the open sets. This turns $\Omega$ into a completely regular topological space. A compactification of $\Omega$ is as usual, a compact Hausdorff space $K$ in which $\Omega$ is topologically embedded and dense. We say that a compactification $K$ of $\Omega$ is an automorphic compactification if every $\pi \in$ $A(\Omega)$ has an extension to a homeomorphism $\bar{\pi}$ of $K$.

Since a totally-ordered set with its order topology is compact if and only if it is lattice-complete we see that $\Omega$ always has an automorphic compactification namely, the lattice completion $\bar{\Omega}$ of $\Omega$.

In this section we exhibit an automorphic compactification $K$ of $\Omega$ for which there is a group of homeomorphisms $\Gamma$ of $K$ such that the maximal tangents for the algebraic tight Riesz order $T_{0}$ on $A(\Omega)$ are in one-one correspondence with the subgroups $\Gamma^{\prime}$ of $\Gamma$ that fix a given point of $K$. The basic idea goes back to Stone (1937), and we proceed in analogy with Gillman and Jerison's procedure (1960) for exhibiting the Stone-Cech compactification of a completely regular space.

For a given topological space $K$ we denote the group of homeomorphisms of $K$ by $\Gamma$, and if $\Gamma^{*}$ is a subgroup of $\Gamma, x$ is a point of $K$, we denote by $\Gamma_{x}^{*}$ the subgroup of $\Gamma^{*}$ consisting of those $\sigma \in \Gamma^{*}$ that fix $x$. That is, $\Gamma_{x}^{*}=$ $\left\{\sigma \in \Gamma^{*}: \sigma(x)=x\right\}$.

We need a preliminary result:

Lemma 9. Let $\mathscr{L}$ be a distributive lattice with minimal element 0 and let $\mathscr{F}$ be a maximal filter of $\mathscr{L}$. If $a \vee b \in \mathscr{F}$ then either $a \in \mathscr{F}$ or $b \in \mathscr{F}$.

Proof. Suppose that $a \notin \mathscr{F}, \quad b \notin \mathscr{F}$. If $c \wedge x \neq 0$ for all $x \in \mathscr{F}$ then $\mathscr{F} \cup\{c\}$ is contained in a filter containing $\mathscr{F}$ so that $c \in \mathscr{F}$. Thus $a \wedge x=0=b \wedge y$ for some $x, y \in \mathscr{F}$, and then $(a \vee b) \wedge(x \wedge y)=(a \wedge x \wedge y) \vee(b \wedge x \wedge y)=0$, so that $a \vee b \notin \mathscr{F}$.

THEOREM 10. There is an automorphic compactification $K$ of $\Omega$ and a subgroup $\Gamma^{*}$ of the group of homeomorphisms of $K$ for which

(1) the maximal tangents for $T_{0}$ are in one-one correspondence with the points of $K$ 
(2) $\Gamma^{*}$ is isomorphic with $A(\Omega)$

(3) if $M_{\xi}$ is the maximal tangent for $T_{0}$ corresponding to $\xi \in K$ then $\Gamma_{\xi}^{*}$ is isomorphic with the normalizer of $M_{\xi}$, and $\Gamma_{\xi}^{*} / M_{\xi}$ is a totally-ordered group.

Proof. We already know, by Theorem 7, that the maximal tangents for $T_{0}$ are in one-one correspondence with the maximal filters of the fixed-point lattice $\Phi(\Omega)$ of $\Omega$. We denote by $K$ the set of maximal filters of $\Phi(\Omega)$. We can furnish $K$ with a topology by taking the sets $\mathscr{U}(\pi)=\{\xi \in K: \operatorname{Fix}(\pi) \notin \xi\}, \pi \in A^{+}(\Omega)$, as basic open sets since, by Lemma $9, \mathcal{U}\left(\pi_{1}\right) \cap \mathcal{U}\left(\pi_{2}\right)=\mathscr{U}\left(\pi_{1} \wedge \pi_{2}\right)$.

$\Omega$ is topologically embedded in $K$ : for $x \in \Omega$ we denote by $\xi(x)$ all those Fix $(\pi) \in \Phi(\Omega)$ with $\pi(x)=x$. Then $\xi(x)$ is plainly a filter of $\Phi(\Omega)$, and if $\xi$ is a filter of $\Phi(\Omega)$ containing $\xi(x)$ then Fix $\left(\pi_{1}\right) \cap \operatorname{Fix}\left(\pi_{2}\right) \neq \square$ for any Fix $\left(\pi_{1}\right) \in \xi$ and Fix $\left(\pi_{2}\right) \in \xi(x)$. In particular, if Fix $\left(\pi_{2}\right)=\{x\}$ we have $x \in \operatorname{Fix}\left(\pi_{1}\right)$ so Fix $\left(\pi_{1}\right) \in \xi(x)$. That is, $\xi(x)$ is a maximal filter of $\Phi(\Omega)$, and the mapping $x \rightarrow \xi(x)$ is plainly one-one. If $U(\pi), \pi \in A^{+}(\Omega)$, is a basic open subset of $K$ then $U(\pi) \cap \Omega=\{x \in \Omega$ : Fix $(\pi) \notin \xi(x)\}=\{x \in \Omega: \pi(x)>x\}=\operatorname{Supp}(\pi)$, which is an open subset of $\Omega$. Conversely, if 0 is an open subset of $\Omega$ then 0 is a union of sets $(-\infty, a),(b, c),(d, \infty)$ which can be taken to be maximal in 0 , so we can define a piecewise linear $\pi \in A^{+}(\Omega)$ with $\operatorname{Supp}(\pi)=0$, and then $0=\mathscr{U}(\pi) \cap \Omega$.

$\Omega$ is dense in $K$ : we take $\xi \in K$, and an open neighbourhood $U(\pi)$ of $\xi$. We have to see that $\mathcal{U}(\pi) \cap \Omega \neq \square$. If $\mathcal{U}(\pi)$ does not meet $\Omega$ then $\operatorname{Supp}(\pi)=\square$ so $\operatorname{Fix}(\pi)=\Omega \in \xi$, and this contradicts $\operatorname{Fix}(\pi) \notin \xi$.

$K$ is Hausdorff: let $\xi_{1}, \xi_{2}$ be maximal filters of $\Phi(\Omega)$. If $\operatorname{Fix}\left(\pi_{1}\right) \in \xi_{2} \backslash \xi_{1}$ then we can find $\operatorname{Fix}\left(\pi_{2}\right) \in \xi_{1}$ satisfying $\operatorname{Fix}\left(\pi_{1}\right) \cap \operatorname{Fix}\left(\pi_{2}\right)=\square$, for otherwise $\xi_{1} \cup\left\{\right.$ Fix $\left.\left(\pi_{1}\right)\right\}$ is contained in a filter larger than the maximal filter $\xi_{1}$. Since $\Omega$ is normal as a topological space (Gillman and Jerison (1960), p. 53) we can find disjoint open sets $O_{1} \supset \operatorname{Fix}\left(\pi_{1}\right), O_{2} \supset \operatorname{Fix}\left(\pi_{2}\right)$. Then there exist $\pi_{1}^{\prime}$, $\pi_{2}^{\prime} \in A^{+}(\Omega)$ with $\operatorname{Fix}\left(\pi_{1}^{\prime}\right)=\Omega \backslash O_{1}, \quad \operatorname{Fix}\left(\pi_{2}^{\prime}\right)=\Omega \backslash O_{2}$. Since $\operatorname{Fix}\left(\pi_{1}^{\prime} \wedge \pi_{2}^{\prime}\right)=$ Fix $\left(\pi_{1}\right) \cup \operatorname{Fix}\left(\pi_{2}^{\prime}\right)=\Omega$, we have $U\left(\pi_{1}^{\prime}\right) \cap \mathscr{U}\left(\pi_{2}^{\prime}\right)=\square$. Further we have Fix $\left(\pi_{1}^{\prime}\right) \notin \xi_{2} \quad$ since $\operatorname{Fix}\left(\pi_{1}^{\prime}\right) \cap \operatorname{Fix}\left(\pi_{1}\right)=\square, \quad$ and $\operatorname{Fix}\left(\pi_{2}^{\prime}\right) \notin \xi_{1} \quad$ since Fix $\left(\pi_{2}^{\prime}\right) \cap \operatorname{Fix}\left(\pi_{2}\right)=\square$. Thus, $\mathcal{U}\left(\pi_{1}^{\prime}\right), \mathcal{U}\left(\pi_{2}^{\prime}\right)$ are disjoint neighbourhoods of $\xi_{2}$, $\xi_{1}$ respectively.

$K$ is compact: we let $\left\{K \backslash \mathcal{U}\left(\pi_{\lambda}\right): \lambda \in \Lambda\right\}$ be a non-empty collection of nonempty basic-closed subsets of $K$ with the finite intersection property. We see that Fix $\left(\pi_{\lambda}\right) \subseteq K \backslash \mathcal{U}\left(\pi_{\lambda}\right)$, and if $K \backslash U(\pi)$ is a basic closed set containing Fix $\left(\pi_{\lambda}\right)$ then Fix $(\pi)=K \backslash \mathcal{U}(\pi) \cap \Omega \supseteq \operatorname{Fix}\left(\pi_{\lambda}\right)$, so that $K \backslash \mathcal{U}\left(\pi_{\lambda}\right) \subseteq K \backslash \mathcal{U}(\pi)$. 
That is, $\overline{\operatorname{Fix}\left(\pi_{\lambda}\right)}=K \backslash U\left(\pi_{\lambda}\right)$ so we see that $\left\{\operatorname{Fix}\left(\pi_{\lambda}\right): \lambda \in \Lambda\right\}$ has the finite intersection property:

$$
\begin{aligned}
\overline{\bigcap_{i=1}^{n} \operatorname{Fix}\left(\pi_{\lambda_{1}}\right)} & =\overline{\operatorname{Fix}\left(\bigvee_{i=1}^{n} \pi_{\lambda_{i}}\right)}=K \backslash \mathcal{U}\left(\sum_{i=1}^{n} \pi_{\lambda_{i}}\right) \\
& =K \backslash \bigcup_{i=1}^{n} \mathcal{U}\left(\pi_{\lambda_{1}}\right)=\bigcap_{i=1}^{n} K \backslash \mathcal{U}\left(\pi_{\lambda_{1}}\right) \neq \square
\end{aligned}
$$

Then, $\left\{\operatorname{Fix}\left(\pi_{\lambda}\right): \lambda \in \Lambda\right\}$ is contained in a maximal filter $\xi$ and so $\xi \in \cap\left\{K \mid \mathcal{U}\left(\pi_{\lambda}\right): \lambda \in \Lambda\right\}$. That is, $K$ is compact.

$K$ is an automorphic compactification: for $\pi \in A(\Omega), \xi \in K$, we define $\bar{\pi}(\xi)=\left\{\pi\left(\operatorname{Fix}\left(\pi^{\prime}\right)\right)\right.$ : $\left.\operatorname{Fix}\left(\pi^{\prime}\right) \in \xi\right\}=\left\{\operatorname{Fix}\left(\pi \pi^{\prime} \pi^{-1}\right)\right.$ : Fix $\left.\left(\pi^{\prime}\right) \in \xi\right\}$. Since $\pi$ is a permutation we see that $\bar{\pi}(\xi)$ is a maximal filter of $\Phi(\Omega)$. Further, $\bar{\pi}$ is then a bijection of $K$ with inverse $\overline{\pi^{-1}}$. If $\mathscr{U}\left(\pi_{1}\right)$ is a basic open set of $K$ then $\bar{\pi} \mathscr{U}\left(\pi_{1}\right)=\mathscr{U}\left(\pi \pi_{1} \pi^{-1}\right)$ so that $\bar{\pi}$ is an open mapping, as is $\bar{\pi}^{-1}$, and therefore a homeomorphism of $K$. Finally, $\bar{\pi}$ restricted to $\Omega$ is $\pi$ since

$$
\begin{aligned}
\bar{\pi}(\xi(x)) & =\left\{\pi\left(\operatorname{Fix}\left(\pi^{\prime}\right)\right): \pi^{\prime}(x)=x\right\}=\left\{\operatorname{Fix}\left(\pi \pi^{\prime} \pi^{-1}\right): \pi^{\prime}(x)=x\right\} \\
& =\left\{\operatorname{Fix}\left(\pi^{\prime \prime}\right): \pi^{\prime \prime} \pi(x)=\pi(x)\right\}=\xi(\pi(x)) .
\end{aligned}
$$

We now observe that since $\overline{\pi_{1} \pi_{2}}=\bar{\pi}_{1} \bar{\pi}_{2}, \Gamma^{*}=\{\bar{\pi}: \pi \in A(\Omega)\}$ is a group of homeomorphisms of $K$ isomorphic to $A(\Omega)$. When we give $\Gamma^{*}$ the identification order i.e. $\sigma \geqslant 1$ in $\Gamma^{*}$ if and only if $\left.\sigma\right|_{\Omega} \geqslant 1$ in $A(\Omega)$, where $\left.\sigma\right|_{\Omega}$ is the restriction of $\sigma$ to $\Omega$, we obtain a lattice ordered group with $\sigma \vee \tau=\overline{\left.\left.\sigma\right|_{\Omega} \vee \tau\right|_{\Omega}}$.

Then the mapping $\pi \rightarrow \bar{\pi}$ is an isomorphism of lattice-ordered groups from $A(\Omega)$ to $\Gamma^{*}$. Recall that for each point $\xi \in K$ we have the following subgroup, $\Gamma_{\xi}^{*}=\left\{\sigma \in \Gamma^{*}: \sigma(\xi)=\xi\right\}$. If $\sigma, \tau \in \Gamma_{\xi}^{*}$ and Fix $(\pi) \in \xi$ then with $\pi_{1}=\left.\sigma\right|_{\Omega}$, and $\pi_{2}=\left.\tau\right|_{\Omega}$ we have

$$
\begin{aligned}
\left(\pi_{1} \vee \pi_{2}\right) \operatorname{Fix}(\pi) & =\operatorname{Fix}\left(\left(\pi_{1} \vee \pi_{2}\right) \pi\left(\pi_{1} \vee \pi_{2}\right)^{-1}\right) \\
& =\operatorname{Fix}\left(\left(\pi_{1} \pi \pi_{1}^{-1} \wedge \pi_{1} \pi \pi_{2}^{-1}\right) \vee\left(\pi_{2} \pi \pi_{1}^{-1} \wedge \pi_{2} \pi \pi_{2}^{-1}\right)\right) \\
& =\left(\operatorname{Fix}\left(\pi_{1} \pi \pi_{1}^{-1}\right) \cup \operatorname{Fix}\left(\pi_{1} \pi \pi_{2}^{-1}\right)\right) \\
& \cap\left(\operatorname{Fix}\left(\pi_{2} \pi \pi_{1}^{-1}\right) \cup \operatorname{Fix}\left(\pi_{2} \pi \pi_{2}^{-1}\right)\right) \\
& \supseteq \operatorname{Fix}\left(\pi_{1} \pi \pi_{1}^{-1}\right) \cap \operatorname{Fix}\left(\pi_{2} \pi \pi_{2}^{-1}\right) \in \xi
\end{aligned}
$$

so $\sigma \vee \tau(\xi) \subseteq \xi$ and thus $\sigma \vee \tau \in \Gamma_{\xi}^{*}$. That is, $\Gamma_{\xi}^{*}$ is a lattice subgroup of $\Gamma^{*}$. The maximal tangent corresponding to the maximal filter $\xi$ is $\boldsymbol{M}_{\xi}=$ $\{\pi \in A(\Omega): \operatorname{Fix}(\pi) \in \xi\}=\{\pi \in A(\Omega): \xi \in \overline{\operatorname{Fix}(\pi)}\}$ and $\sigma=\overline{\left.\pi\right|_{\Omega}} \in \Gamma_{\xi}^{*}$ if and only if $\left\{\operatorname{Fix}\left(\pi \pi^{\prime} \pi^{-1}\right)\right.$ : $\left.\operatorname{Fix}(\pi) \in \xi\right\}=\{\operatorname{Fix}(\pi): \pi \in \xi\}$. That is, $\sigma=\left.\pi\right|_{\Omega} \in \Gamma_{\xi}^{*}$ is and only if $\pi$ is in the normalizer of $M_{\xi}$. Thus $\Gamma_{\xi}^{*}$ is isomorphic with a lattice subgroup which is the normalizer of $M_{\xi}$ in $A(\Omega)$ so that, by an abuse of 
notation, $M_{\xi}$ is a normal prime subgroup of $\Gamma_{\xi}^{*}$ and $\Gamma_{\xi}^{*} / M_{\xi}$ is a totally ordered group.

REMARK. If $\xi=\xi(x)$ for some $x \in \Omega$ then $M_{\xi(x)}=P_{x}=$ $\{\pi \in A(\Omega): \pi(x)=x\}$ is easily seen to be its own normalizer and therefore $\Gamma_{\xi}^{*} / M_{\xi}=(0)$ in this case.

We can now adopt a similar procedure for an arbitrary algebraic tight Riesz order $T$ on $A(\Omega)$. Specifically, we denote by $K(T)$ the set of filters of the fixed-point lattice $\Phi(\Omega)$ that are maximal with respect to not meeting the $A(\Omega)$-invariant ideal $f(T)$ of $A(\Omega)$ corresponding to $T$. The following remark then says that these filters are prime, so we can take the sets $U(\pi)=$ $\{\xi \in K(T)$ : Fix $(\pi) \notin \xi\} \subseteq K(T)$ as a join-base for the open sets for a topology on $K(T)$.

REMARK. As a more general form of Lemma 9 we have the following which is the dual of a well-known result of Stone.

Let $\mathscr{L}$ be a distributive lattice and $I \subseteq \mathscr{L}$ a proper ideal of $\mathscr{L}$. If $\mathscr{F}$ is a filter of $\mathscr{L}$ maximal with respect to not meeting $I$ then $a \vee b \in \mathscr{F}$ implies $a \in \mathscr{F}$ or $b \in \mathscr{F}$.

We regard the algebraic tight Riesz orders as forming a lattice of subsets of $A(\Omega)$ by conveniently regarding $A^{+}(\Omega)$ as an algebraic tight Riesz order. This lattice of algebraic tight Riesz orders on $A(\Omega)$ is lattice-isomorphic with the lattice $\mathscr{T}$ of $A(\Omega)$-invariant ideals of $\Phi(\Omega)$.

THEOREM 11. For each algebraic tight Riesz order $T$ of $A(\Omega), K(T)$ is a compact Hausdorff space, and $T \rightarrow K(T)$ is the object function of a contravariant functor from $\mathscr{T}$ into the category of compact Hausdorff spaces and continuous maps.

Proof. The proof that $K(T)$ is compact Hausdorff is as in Theorem 10 . Suppose that $T_{1}, T_{2}$ are algebraic tight Riesz orders and $T_{1} \subseteq T_{2}$. If $M$ is a maximal tangent for $T_{2}$ then, since the prime subgroups lying above a prime subgroup are totally-ordered by inclusion, there is a unique maximal tangent $M^{\prime}$ for $T_{1}$ containing $M$. This provides us with a map, which we denote by $m$, from $K\left(T_{2}\right)$ into $K\left(T_{1}\right)$. Given a basic open subset $u_{1}(\pi)=$ $\left\{\xi \in K\left(T_{1}\right):\right.$ Fix $\left.(\pi) \notin \xi\right\}$ we have to see that $\mathscr{S}=\left\{\xi \in K\left(T_{2}\right): m(\xi) \in \mathcal{U}_{1}(\pi)\right\}$ is open in $K\left(T_{2}\right)$, and we do this by seeing that $K\left(T_{2}\right) \backslash \mathscr{S}$ is compact. Let $\left\{K\left(T_{2}\right) \backslash \mathscr{U}_{2}\left(\pi_{\lambda}\right) \cap K\left(T_{2}\right) \backslash \mathscr{S}: \lambda \in \Lambda\right\}$ be a collection of basic closed subsets of $K\left(T_{2}\right) \backslash \mathscr{S}$ with the finite intersection property, where $\mathscr{U}_{2}(\pi)=$ $\left\{\xi \in K\left(T_{2}\right): \operatorname{Fix}(\pi) \notin \xi\right\}$ for $\pi \in A(\Omega)$. Then for each finite subset $\left\{\lambda_{1}, \cdots, \lambda_{n}\right\}$ of $\Lambda$ we have $\mathscr{P} \cup \mathscr{U}_{2}\left(\pi_{\lambda_{1}}\right) \cup \cdots \cup \mathcal{U}_{2}\left(\pi_{\lambda_{n}}\right) \neq K\left(T_{2}\right)$. If Fix $(\pi) \cap \operatorname{Fix}\left(\pi_{\lambda_{1}}\right) \cap \cdots \cap$ $\operatorname{Fix}\left(\pi_{\lambda_{n}}\right) \in f\left(T_{1}\right)$, for some finite subset $\left\{\lambda_{1}, \cdots, \lambda_{n}\right\}$ of $\Lambda$, then $\mathscr{U}_{1}(\pi) \cup$ $\mathcal{U}_{1}\left(\pi_{\lambda_{1}}\right) \cup \cdots \cup \mathcal{U}_{1}\left(\pi_{\lambda_{n}}\right)=K\left(T_{1}\right)$. We know, by assumption, that there is a 
$\xi \in K\left(T_{2}\right)$ such that $\xi \notin \mathscr{P}$ and $\xi \notin \mathscr{U}_{2}\left(\pi_{\lambda_{i}}\right)(i=1, \cdots, n)$. Then $m(\xi) \notin \mathscr{U}_{1}(\pi)$ so that $m(\xi) \in \mathscr{U}_{1}\left(\pi_{\lambda_{i}}\right)$ for some $i$, which implies $\xi \in \mathcal{U}_{2}\left(\pi_{\lambda_{1}}\right)$ - a contradiction. Thus, for every finite subset $\left\{\lambda_{1}, \cdots, \lambda_{n}\right\}$ of $\Lambda$, Fix $(\pi) \cap \operatorname{Fix}\left(\pi_{\lambda_{1}}\right) \cap \cdots \cap$ Fix $\left(\pi_{\lambda_{n}}\right) \notin f\left(T_{1}\right)$. Similarly, for every finite subset $\left\{\lambda_{1}, \cdots, \lambda_{n}\right\}$ of $\Lambda$, Fix $\left(\pi_{\lambda_{1}}\right) \cap$ ... Fix $\left(\pi_{\lambda_{n}}\right) \notin f\left(T_{2}\right)$. Hence, there is a $\xi \in K\left(T_{2}\right)$ containing all Fix $\left(\pi_{\lambda}\right): \lambda \in$ $\Lambda$, and a $\xi^{\prime} \in K\left(T_{1}\right)$ containing $\xi$ and Fix $(\pi)$. Then $m(\xi)=\xi^{\prime}$ so we have $\xi \notin \mathscr{S}$ and $\xi \notin U_{2}\left(\pi_{\lambda}\right)$ for all $\lambda \in \Lambda$. That is, $K\left(T_{2}\right) \backslash \mathscr{S}$ is compact. This gives us an extension of $T \rightarrow K(T)$ to arrows and provides us with a (contravariant) functor.

We denote by $\Gamma(T)$ the group of homeomorphisms of $K(T)$.

Proposition 12. For each algebraic tight Riesz order $T$ of $A(\Omega)$ there is a homomorphism $\delta_{T}: A(\Omega) \rightarrow \Gamma(T)$ for which $\operatorname{Ker}\left(\delta_{T}\right)$ is a normal sublattice subgroup of $A(\Omega)$. Further if we denote $\operatorname{Im}\left(\delta_{T}\right)$ by $\Gamma^{*}$ then, for each $\xi \in$ $K(T), \Gamma_{\xi}^{*}=\left\{\sigma \in \Gamma^{*}: \sigma(\xi)=\xi\right\}$ is the image under $\delta_{T}$ of the normalizer of the maximal tangent for $T$ corresponding to $\xi$.

Proof. We define $\delta_{T}$ by $\delta_{T}(\pi)(\xi)=\pi \cdot \xi=\{\pi(F): F \in \xi\}$. Because $f(T)$ is an $A(\Omega)$-invariant ideal of $\Phi(\Omega)$ we see, as in Theorem 10 , that for each $\pi \in A(\Omega), \delta_{T}(\pi)$ is a homeomorphism of $K(T)$. Further, $\delta_{T}: A(\Omega) \rightarrow \Gamma(T)$ is clearly a homomorphism, and calculations we have already seen in Theorem 11 show that $\operatorname{Ker}\left(\delta_{T}\right)=\{\pi \in A(\Omega): \pi \cdot \xi \subseteq \xi$ for all $\xi \in K(T)\}$ is a sublattice of $A(\Omega)$, and that the last statement of the proposition is valid.

\section{Groups acting on lattices}

We say that a group $G$ acts on a lattice $L$ if there is a map $(g, x) \rightarrow g x ; G \times$ $L \rightarrow L$ satisfying

(1) $g(x \vee y)=(g x) \vee(g y)$

(2) $g(x \wedge y)=(g x) \wedge(g y)$

(3) $(g h) x=g(h x)$

(4) $1 x=x$.

We say, in addition, that $G$ acts properly on $L$ if $g x=x$, for all $x \in L$, implies $g=1$. In other words, $G$ acts properly on $L$ precisely when $G$ is isomorphic with a subgroup of the group of lattice automorphisms of $L$.

A subset $S$ of $L$ is $G$-invariant if $x \in S, g \in G$ implies $g x \in S$. An element $x \in L$ is $G$-invariant if the singleton set $\{x\}$ is $G$-invariant. The class of $G$-invariant ideals containing a given $G$-invariant ideal $I$ is inductive, so there is a maximal $G$-invariant ideal containing $I$.

For the remainder of this section we will assume that $L$ is distributive.

Proposition 13. Let $M$ be a maximal (proper) G-invariant ideal of $L$. If $a \notin M, b \notin M$ then there is $a g \in G$ for which $a \wedge g b \notin M$. 
Proof. Suppose that $a \wedge g b \in M$ for all $g \in G$. Let $N=$ $\{x \in L: x \leqq y \vee g b$ for some $y \in M, g \in G\}$. Then $N$ is clearly a $G$-invariant ideal which properly contains $M$, since $b \in N$, and is properly contained in $L$, since $a \notin N$. This is a contradiction.

A dual result is valid for $G$-invariant filters of $L$. We say that a $G$-invariant ideal $I$ of $L$ is $G$-prime if it satisfies the conclusion of the proposition, that is, if $a \wedge g b \in I$ for all $g \in G$, implies $a \in I$ or $b \in I$.

A standard argument now gives the following result.

Proposition 14. Let I be a G-invariant ideal of $L$. If $x \notin I$ then any $G$-invariant ideal $M$ of $L$, maximal with respect to containing $I$ but not $x$, is $G$-prime. In particular, every $G$-invariant ideal of $L$ is an intersection of $G$-prime G-invariant ideals.

For a subset $S$ of $L$ we define Orth $(S)=\{g \in G: a \wedge b \in S$ implies $a \wedge g$ $b \in S\}$.

Proposition 15. If $S$ is a $G$-invariant subset of $L$ then Orth $(S)$ is a normal subgroup of $G$. If $I$ is a $G$-invariant ideal of $L$ then $I$ is prime if and only if $I$ is $G$-prime and Orth $(I)=G$.

Proof. If $g \in$ Orth $(S)$, and $a \wedge b \in S$ then $b \wedge a \in S$ so that $b \wedge g a \in S$. Then $g^{-1} b \wedge a=g^{-1}(b \wedge g a) \in S$ so $g^{-1} \in$ Orth $(S)$. If, in addition, $h \in G$ then $h^{-1} a \wedge h^{-1} b=h^{-1}(a \wedge b) \in S$ so that $a \wedge h g h^{-1} b=h\left(h^{-1} a \wedge g h^{-1} b\right) \in S$. Since Orth $(S)$ is clearly a semigroup we see that Orth $(S)$ is a normal subgroup of $G$. The second statement is clear.

For a $G$-prime $G$-invariant ideal $I$ of $L$ the group $G / O r t h(I)$ gives us a group-theoretic measure of the extent to which $I$ fails to be prime. We have a dual procedure in the case of $G$-invariant filters $F$ of $L$, for which we define $\operatorname{Orth}^{\circ}(F)=\{g \in G: a \vee b \in F$ implies $a \vee g b \in F\}$.

We now define $\operatorname{Spec}_{G}(L)$ to be the set of $G$-prime $G$-invariant ideals of $L$ furnished with the topology that has the sets $\mathscr{U}(x)=\{I \subseteq L: I$ is a $G$-prime $G$-invariant ideal and $x \notin I\}, x \in L$, as sub-basic open sets. We see that $\{U(x): x \in L\}$ is in fact a join-base for the open sets, for if $I \in \mathcal{U}(x) \cap \mathcal{U}(y)$ then $x \notin I, \quad y \notin I$ so that $x \wedge g y \notin I$ for some $g \in G$, and thus $I \in \mathcal{U}(x \wedge g y) \subseteq$ $\mathcal{U}(x) \cap \mathcal{U}(y)$. A standard argument now shows that $\operatorname{Spec}_{G}(L)$ is a locallycompact $T_{0}$ space which is compact if and only if $L$ has a greatest element.

We remark finally on a partition of the maximal $A(\Omega)$-invariant filters of the lattice $2^{\Omega^{*}}$ where, as usual, $\Omega$ is a totally-ordered field and where $\Omega^{*}$ is $\Omega$ with the discrete order. The action of $A(\Omega)$ on $2^{\Omega^{*}}$ is given by $(\pi, \chi) \rightarrow \chi \cdot \pi^{-1}$.

Proposition 16. If $\mathscr{F}$ is a maximal $A(\Omega)$-invariant filter of $2^{\mathrm{N}^{*}}$ then either $[0, \infty) \in \mathscr{F}$ or $(-\infty, 0] \in \mathscr{F}$ but not both. 
Proof. Firstly we suppose that $\mathscr{G}$ is an $A(\Omega)$-invariant filter and that $[0, \infty) \cap S \neq \square$ for all $S \in \mathscr{G}$. If $x \leqq 0$ in $\Omega$ then $[x, \infty) \supseteq[0, \infty)$ so $[x, \infty) \cap S \neq \square$ for all $S \in \mathscr{G}$. If $x>0$ we can find $\pi \in A(\Omega)$ such that $\pi(x)=0$ and then $[x, \infty) \cap S=\square$ for some $S \in \mathscr{G}$ implies $\square=[\pi(x), \infty) \cap \pi(S)=[0, \infty) \cap \pi(S)$, which contradicts our assumption since $\pi(S) \in \mathscr{G}$. We see immediately, since $\mathscr{F}$ is maximal, that if $[0, \infty) \in \mathscr{F}$ then $[x, \infty) \in \mathscr{F}$ for all $x \in \Omega$, and a similar conclusion holds for the intervals $(-\infty, x], x \in \Omega$. Thus the cases $(-\infty, 0] \in \mathscr{F}$, $[0, \infty) \in \mathscr{F}$ are mutually exclusive. Now suppose that $(-\infty, 0] \notin \mathscr{F}$ and $[0, \infty) \notin \mathscr{F}$. Then, $[0, \infty) \cap F_{1}=\square=(-\infty, 0] \cap F_{2}$ for some $F_{1}, F_{2} \in \mathscr{F}$ so that

$$
\begin{aligned}
\square & =\left([0, \infty) \cap F_{1}\right) \cup\left((-\infty, 0] \cap F_{2}\right) \\
& =\left([0, \infty) \cup F_{2}\right) \cap\left(F_{1} \cup(-\infty, 0]\right) \cap\left(F_{1} \cup F_{2}\right) \in \mathscr{F}
\end{aligned}
$$

which is a contradiction.

\section{Tight Riesz orders and first-order properties}

In this section we relate tight Riesz orders on $A(\Omega)$ to filters on the discretely ordered set $\Omega$ and thereby to subsets of $\Omega$.

We write $\Omega^{*}$ for $\Omega$ with the discrete order (i.e. $x \leqq y$ if and only if $x=y$ ). The lattice $2^{\Omega^{*}}$ is isomorphic with the lattice of all subsets of $\Omega$, and the group $A(\Omega)$ acts on the lattice $2^{\Omega^{*}}$.

THEOREM 17. There is an adjunction from the set of tight Riesz orders on $A(\Omega)$, ordered by inclusion, to the set of $A(\Omega)$-invariant filters of $2^{\Omega^{*}}$, ordered by inclusion, such that the algebras for this adjunction are precisely the algebraic tight Riesz orders.

Proof. We describe the adjunction: the proof that it is an adjunction and that the algebras are just the algebraic tight Riesz orders follows as in Theorem 4. If $T$ is a tight Riesz order then $\{\operatorname{Supp}(\pi): \pi \in T\}$ is an $A(\Omega)$-invariant filterbase of $2^{\boldsymbol{N}^{*}}$ since $\pi_{1}(\operatorname{Supp}(\pi))=\operatorname{Supp}\left(\pi_{1} \pi \pi_{1}^{-1}\right)$ for all $\pi \geqq 1, \pi_{1} \in A(\Omega)$. We denote by $f(T)$ the $A(\Omega)$-invariant filter of $2^{\Omega^{*}}$ generated by $\{\operatorname{Supp}(\pi): \pi \in$ $T$ \}. On the other hand, if $\mathscr{F}$ is an $A(\Omega)$-invariant filter of $2^{\alpha^{*}}$ then $g(\mathscr{F})=$ $\left\{\pi \in A^{+}(\Omega)\right.$ : $\left.\operatorname{Supp}(\pi) \in \mathscr{F}\right\}$ is a tight Riesz order on $A(\Omega)$, and $f$ is a leftadjoint for $g$.

We say that a subset $F$ of $\Omega$ is an ENBH set (for "eventually no big holes") if $\pi_{1}(F) \cap \cdots \cap \pi_{n}(F) \neq \square$ for every finite subset $\left\{\pi_{1}, \cdots, \pi_{n}\right\}$ of $A(\Omega)$, and we denote the set of all ENBH subsets of $\Omega$ by $\mathscr{L}(\Omega)$. Thus, ordered by inclusion, $\mathscr{L}(\Omega)$ contains $\Omega$, does not contain $\square$, satisfies the property that if $F \in \mathscr{L}(\Omega)$ and $G \supseteq F$ then $G \in \mathscr{L}(\Omega)$. 
If $F$ is an ENBH set then $\{\pi(F): \pi \in A(\Omega)\}$ is a filterbase of $2^{\Omega^{*}}$ and the filter generated by this filterbase is an $A(\Omega)$-invariant filter containing $F$. Conversely if $\mathscr{F}$ is an $A(\Omega)$-invariant filter of $2^{\Omega^{*}}$ and $F \in \mathscr{F}$ then $F$ is an ENBH set.

We denote by $\mathscr{W}$ the set of subsets of $\Omega$ determined in the following way: $W \in \mathscr{W}$ if and only if there is a property $\mathscr{P}$ of the theory of totally-ordered sets, such that

(1) $\mathscr{P}$ is preserved under permutations in $\Omega$. That is, if $S \subseteq \Omega$ has property $\mathscr{P}$ and if $\pi \in A(\Omega)$ then $\pi(S)$ has property $\mathscr{P}$.

(2) $\mathscr{P}$ is preserved under finite unions in $\Omega$. That is, if $\left\{S_{1}, \cdots, S_{n}\right\}$ is a finite collection of subsets of $\Omega$ such that each $S_{i}$ has property $\mathscr{P}$ then $S_{1} \cup \cdots \cup S_{n}$ has property $\mathscr{P}$.

(3) $\Omega$ does not have property $\mathscr{P}$.

(4) $W$ does have property $\mathscr{P}$.

We denote by $\mathscr{W}^{*}$ the set of subsets $W \subseteq \Omega$ which are determined by a first-order property $\mathscr{P}$ satisfying (2), (3), (4). We regard $\mathscr{W}$ and $W^{*}$ as ordered by inclusion.

THEOREM 18. There is a functor from $W$ into the lattice of algebraic tight Riesz orders on $A(\Omega)$.

Proof. If $W \in W$ and $\left\{\pi_{1}, \cdots, \pi_{n}\right\}$ is a finite subset of $A(\Omega)$ then $\pi_{1}(W) \cup \cdots \cup \pi_{n}(W) \neq \Omega$ so that $F=\Omega \backslash W$ is an ENBH set. We denote by $T(W)$ the algebraic tight Riesz order on $A(\Omega)$ corresponding to the $A(\Omega)$ invariant filter of $2^{\Omega^{*}}$ generated by the filterbase $\{\pi(F): \pi \in A(\Omega)\}$. Thus, $T(W)=\left\{\pi \in A^{+}(\Omega): \operatorname{Fix}(\pi) \subseteq \pi_{1}(W) \cup \cdots \cup \pi_{n}(W)\right.$ for some $\left.\pi_{i} \in A(\Omega)\right\}$, so that $W \rightarrow T(W)$ is a functor from $W$ into the lattice of algebraic tight Riesz orders on $A(\Omega)$.

COROLLARY 19. There is a functor from $W^{*}$ into the lattice of algebraic tight Riesz orders on $A(\Omega)$.

We note also that there is a way of obtaining an algebraic tight Riesz order from the collection of all subsets of $\Omega$ satisfying a property for which (1)-(3) holds. In fact let $\mathscr{P}$ be such a property. Then the set $\{\pi(F): \pi \in A(\Omega), F=\Omega \backslash S$ for some $S \subseteq \Omega$ satisfying $\mathscr{P}\}$ is an $A(\Omega)$-invariant filterbase of $2^{\Omega^{*}}$, and we denote the corresponding algebraic tight Riesz order by $T(\mathscr{P})$.

Then the assignment $W \rightarrow T(W)$ gives us an algebraic tight Riesz order for each subset $W$ of $\Omega$ satisfying a property $\mathscr{P}$ for which (1)-(4) holds, whilst the assignments $\mathscr{P} \rightarrow T(\mathscr{P})$ gives us an algebraic tight Riesz order for each property $\mathscr{P}$ for which (1)-(3) holds. If $W$ is a subset of $\Omega$ satisfying $\mathscr{P}$ then $T(W) \subseteq T(\mathscr{P})$. 
In fact then, the ENBH sets are only instances or models of the properties. For this reason, the following section is written in terms of properties and not specific models.

\section{Automorphisms of the real field}

In this section we consider some of the previous results and concepts for $\Omega=R$, the unique complete ordered field. One of the first things to note (and this is true in general) is the symmetry of the lattice of algebraic tight Riesz orders (recall that we are conveniently regarding $A^{+}(\Omega)$ as an algebraic tight Riesz order).

Proposition 20. The lattice $\mathscr{T}$ of algebraic tight Riesz orders on $A(\Omega)$ has an automorphism of order 2.

Proof. We denote by $\nu$ the anti-automorphism $x \rightarrow-x$ of $\Omega$. If $T$ is an algebraic tight Riesz order on $A(\Omega)$ then $\{\nu(F): F \in f(T)\}$ is an $A(\Omega)$-invariant ideal of $\Phi(\Omega)$, and we denote the corresponding algebraic tight Riesz order by $T^{*}$. Then $T \rightarrow T^{*}$ is an automorphism of $\mathscr{T}$ of order 2 .

By exhibiting subsets $W$ of $R$ satisfying $\pi_{1}(W) \cup \cdots \cup \pi_{n}(W) \neq R$ for all finite subsets $\left\{\pi_{1}, \cdots, \pi_{n}\right\}$ of $A(R)$, we can list algebraic tight Riesz orders

$$
\begin{array}{r}
T(W)=\left\{\pi \in A^{+}(R): F i x(\pi) \subseteq \pi_{1}(W) \cup \cdots \cup \pi_{n}(W),\right. \\
\left.\quad \text { for some finite subset }\left\{\pi_{1}, \cdots, \pi_{n}\right\} \text { of } A(R)\right\}
\end{array}
$$

and similarly by listing properties $\mathscr{P}$ of the theory of totally-ordered sets that are not satisfied by $R$, but are preseved, in $R$, by permutations and finite unions, we can list algebraic tight Riesz orders

$$
\begin{aligned}
T(\mathscr{P})= & \left\{\pi \in A^{+}(R): F i x(\pi) \subseteq \pi_{1}(S) \cup \cdots \cup \pi_{n}(S),\right. \text { for } \\
& \text { some finite subset }\left\{\pi_{1}, \cdots, \pi_{n}\right\} \text { of } A(\Omega), \text { and } \\
& \text { some } S \subseteq R \text { satisfying } \mathscr{P}\} .
\end{aligned}
$$

We do this, in part, in the following table where we also state whether the corresponding $T(\mathscr{P})$ are $A(R)$-prime (or prime), and list the normal subgroups Orth $(f(T(\mathscr{P})))$ of $A(R)$. Recall that a tight Riesz order $T$ is prime if given $\pi_{1}, \pi_{2} \in A^{+}(R)$ such that $\pi_{1} \vee \pi_{2} \in T$ then either $\pi_{1} \in T$ or $\pi_{2} \in T$. Similarly $T$ is $A(R)$-prime if given $\pi_{1}, \pi_{2} \in A^{+}(R)$ such that $\pi_{1} \vee \pi \pi_{2} \pi^{-1} \in T$ for all $\pi \in A(R)$ then either $\pi_{1} \in T$ or $\pi_{2} \in T$. We also have a more straightforward definition of Orth $(T)$ for $T$ a tight Riesz order on $A(R)$, namely

$$
\operatorname{Orth}(T)=\left\{\pi \in A(R): \pi_{1} \vee \pi_{2} \in T \text { implies } \pi_{1} \vee \pi \pi_{2} \pi^{-1} \in T\right\}
$$

(Note that $\operatorname{Orth}(T)=\operatorname{Orth}(f(T)))$. 
Algebraic tight Riesz

Defining

Primality

Orth $(T(\mathscr{P}))$

order, $T(\mathscr{P})$

Property

of $T(\mathscr{P})$

$\begin{array}{cccc}T_{\alpha} & \text { lower section } & A(R) \text {-prime } & A \\ T_{\beta} & \text { upper section } & A(R) \text {-prime } & B \\ T_{w} & \text { well-ordered } & A(R) \text {-prime } & \langle 1\rangle \\ T_{\gamma} & \text { finite } & - & \langle 1\rangle \\ T_{0} & \text { empty } & A(R) \text {-prime } & \langle 1\rangle\end{array}$

where

$$
A=\{\pi \in A(R): \text { for some } y \in R, \pi(x)=x \text { for } x \geqq y\}
$$

and

$$
B=\{\pi \in A(R) \text { : for some } y \in R, \pi(x)=x \text { for } x \leqq y\}
$$

We note (c.f. Holland (1963) p. 406) that $A, B$ and $C=A \cap B$ are the only proper normal subgroups of $A(R)$.

We now show that $T_{\alpha}$ is $A(R)$-prime and Orth $\left(T_{\alpha}\right)=A$. Suppose that $\pi_{1}, \pi_{2} \in A^{+}(R) \backslash T_{\alpha}$ and $\pi_{1} \vee \pi \pi_{2} \pi^{-1} \in T_{\alpha}$ for all $\pi \in A(R)$. Then for each $y \in R$ there exist $z_{1}, z_{2} \geqq y$ for which $\pi_{1}\left(z_{1}\right)=z_{1}$ and $\pi_{1}\left(z_{2}\right)=z_{2}$. We choose $\pi \in A(R)$ such that $\pi\left(z_{2}\right)=z_{1}$, and then $\pi_{1} \vee \pi \pi_{2} \pi^{-1}\left(z_{1}\right)=z_{1}$. Thus, for each $y \in R$, $\pi_{1} \vee \pi \pi_{2} \pi^{-1}$ has a fixed point larger than $y$ which contradicts $\pi_{1} \vee \pi \pi_{2} \pi^{-1} \in T_{\alpha}$. Thus, $T_{\alpha}$ is $A(R)$-prime, and clearly not prime. We have immediately $\operatorname{Orth}\left(T_{\alpha}\right) \neq A(R)$. If $\pi \in A$ and $\pi_{1} \vee \pi_{2} \in T_{\alpha}$ then there exist $y, y^{\prime} \in R$ such that $\pi(x)=x$ for $x \geqq y$ and $\pi_{1} \vee \pi_{2}(x)>x$ for $x \geqq y^{\prime}$. Then with $y^{\prime \prime}=$ $\max \left\{y, y^{\prime}\right\}$ we have $\pi_{1} \vee \pi \pi_{2} \pi^{-1}(x)>x$ for $x \geqq y^{\prime \prime}$, so that $\pi_{1} \vee \pi \pi_{2} \pi^{-1} \in T_{\alpha}$. Thus $A \subseteq \operatorname{Orth}\left(T_{\alpha}\right) \neq A(R)$, so $A=\operatorname{Orth}\left(T_{\alpha}\right)$. A similar srgument gives us the corresponding statement for $T_{\beta}$.

The tight Riesz order $T_{w}$ is $A(R)$-prime since $\pi \in A^{+}(R) \backslash T_{w}$ if and only if Fix $(\pi)$ contains a subset similar to the inversely-ordered natural numbers, so given $\pi_{1}, \pi_{2} \in A^{+}(R) \backslash T_{w}$ we can find $\pi \in A(R)$ such that Fix $\left(\pi_{1} \vee \pi \pi_{2} \pi^{-1}\right)$ contains a subset of $R$ similar to the inversely-ordered natural numbers.

The tight Riesz order $T_{\gamma}$ is not $A(R)$-prime for if we let $\pi_{1}, \pi_{2}$ be piecewise linear elements of $A^{+}(R)$ with Fix $\left(\pi_{1}\right)=\{0\} \cup\{1 / n: n=1,2, \cdots\}$ and Fix $\left(\pi_{2}\right)=\{2,3,4, \cdots\}$ then $\pi_{1}, \pi_{2} \notin T_{\gamma}$ but $\pi_{1} \vee \pi \pi_{2} \pi^{-1} \in T_{y}$ for all $\pi \in A(R)$.

The transitivity of $A(R)$ gives that $T_{0}$ is $A(R)$-prime, so it remains to see that $\operatorname{Orth}\left(T_{w}\right)=\operatorname{Orth}\left(T_{\gamma}\right)=\operatorname{Orth}\left(T_{0}\right)=\langle 1\rangle$. We do this in the case of $T_{0}$, for instance, by choosing piecewise linear $\pi_{1}, \pi_{2} \in A^{+}(R)$ with $\pi_{1} \vee \pi_{2} \in T_{0}$ and a piecewise linear $\pi \in C$ such that $\pi_{1} \vee \pi \pi_{2} \pi^{-1} \notin T_{0}$. Then $C \not \subset \operatorname{Orth}\left(T_{0}\right)$ so $\operatorname{Orth}\left(T_{0}\right)=\langle 1\rangle$. A similar procedure works for $T_{w}$ and $T_{v}$. 
Lemma 21. Any tight Riesz order on $A(R)$ obtained as an intersection of two distinct tight Riesz orders on $A(R)$ fails to be $A(R)$-prime.

Proof. Let $T_{1}$ and $T_{2}$ be tight Riesz orders, such that $T=T_{1} \cap T_{2}$ is a tight Riesz order distinct from both $T_{1}$ and $T_{2}$. Choose $\pi_{1} \in T_{1} \mid T_{2}$ and $\pi_{2} \in T_{2} \backslash T_{1}$. Then $\pi \pi_{2} \pi^{-1} \in T_{2} \backslash T_{1}$ for all $\pi \in A(R)$, since $\pi \pi_{2} \pi^{-1} \in T_{1}$ implies $\pi_{2} \in T_{1}\left(T_{1}\right.$ normal). However $\pi_{1} \vee \pi \pi_{2} \pi^{-1} \geqslant \pi_{1}$ implies $\pi_{1} \vee \pi \pi_{2} \pi^{-1} \in T_{1}\left(T_{1}\right.$ a dual ideal) and similarly $\pi_{1} \vee \pi \pi_{2} \pi^{-1} \in T_{2}$, i.e. $\pi_{1} \vee \pi \pi_{2} \pi^{-1} \in T$ for all $\pi \in A(R)$, whence $T$ is not $A(R)$ prime.

It now remains for us to find Orth $\left(T_{\alpha \beta}\right)$. In doing this we use arguments similar to those used previously in conjunction with the following result.

THEOREM 22. Let $T_{1}$ and $T_{2}$ be tight Riesz orders on $A(R)$ with $T_{1} \cap T_{2}$ a further tight Riesz order, then

$$
\operatorname{Orth}\left(T_{1} \cap T_{2}\right) \supseteq \operatorname{Orth}\left(T_{1}\right) \cap \operatorname{Orth}\left(T_{2}\right) .
$$

Proof. We have $\pi_{1} \vee \pi_{2} \in T_{1} \cap T_{2} \Rightarrow \pi_{1} \vee \pi_{2} \in T_{1} \Rightarrow \pi_{1} \vee \pi \pi_{2} \pi^{-1} \in T_{1}$ for $\pi \in \operatorname{Orth}\left(T_{1}\right)$. Similarly, $\pi_{1} \vee \pi_{2} \in T_{1} \cap T_{2} \Rightarrow \pi_{1} \vee \pi \pi_{2} \pi^{-1} \in T_{2}$ for $\pi \in \operatorname{Orth}\left(T_{2}\right)$. Thus $\quad \pi_{1} \vee \pi_{2} \in T_{1} \cap T_{2} \Rightarrow \pi_{2} \vee \pi \pi_{2} \pi^{-1} \in T_{1} \cap T_{2} \quad$ for $\pi \in \operatorname{Orth}\left(T_{1}\right) \cap \operatorname{Orth}\left(T_{2}\right)$.

We complete the table of results as follows:

Algebraic tight Riesz order $T$

$T_{\alpha \beta}$
$\operatorname{Orth}(T)$

C

We conclude with some questions that we could not answer satisfactorily:

(1) Let $K$ be a Hausdorff topological space with a subset $T$ satisfying

(i) $T$ is totally-ordered and order-dense

(ii) the induced topology on $T$ is the order topology.

(iii) $T$ is dense in $K$.

When can the order on $T$ be extended to a total order on $K$ such that the given topology on $K$ is the order topology?

(2) Does the functor $T \rightarrow K(T)$ from the lattice of algebraic tight Riesz orders into the category of compact Hausdorff spaces preserve finite products and coproducts?

(3) Is the mapping $T \rightarrow$ Orth ( $T$ ) from the lattice of algebraic tight Riesz orders into the lattice of normal subgroups of $A(\Omega)$ a functor? If so, does it preserve finite products and coproducts? 
(4) Is there an element-wise characterization of minimal $G$-prime $G$-invariant ideals of a distributive lattice $L$ on which a group $G$ acts?

(5) If $G$ is a group acting on a distributive lattice $L$, are the $G$-prime $G$-invariant ideals of $L$ precisely the finitely-meet-irreducible elements of the lattice of $G$-invariant ideals of $L$ ?

\section{References}

L. Gillman and M. Jerison (1960), 'Rings of continuous functions', Van Nostrand, Princeton.

Charles Holland (1963), 'The lattice-ordered group of automorphisms of an ordered set', Mich. Math. J. 10, 399-408.

Saunders Mac Lane (1971), 'Categories for the working mathematician', Springer-Verlag, New York.

Stephen H. McCleary (1969), 'The closed prime subgroups of certain ordered permutation groups', Pac. J. Math. Vol 31, 3, 745-753.

J. B. Miller (1973), 'Quotient groups and realization of tight Riesz groups', J. Austral. Math. Soc. 16, 416-430.

N. R. Reilly (1973), 'Compatible tight Riesz orders and prime subgroups', Glasgow Math. J. 14, 145-160.

N. R. Reilly (to appear), 'Representations of ordered groups with compatible tight Riesz orders', $J$. Austral. Math. Soc.

M. H. Stone (1937), 'Applications of the theory of Boolean Rings to general topology', Trans. Amer. Math. Soc., 41, 375-481.

A. Wirth (1973), 'Compatible tight Riesz orders', J. Austral. Math. Soc. 15, 105-111.

Department of Mathematics

La Trobe University

Victoria, 3083

Australia. 